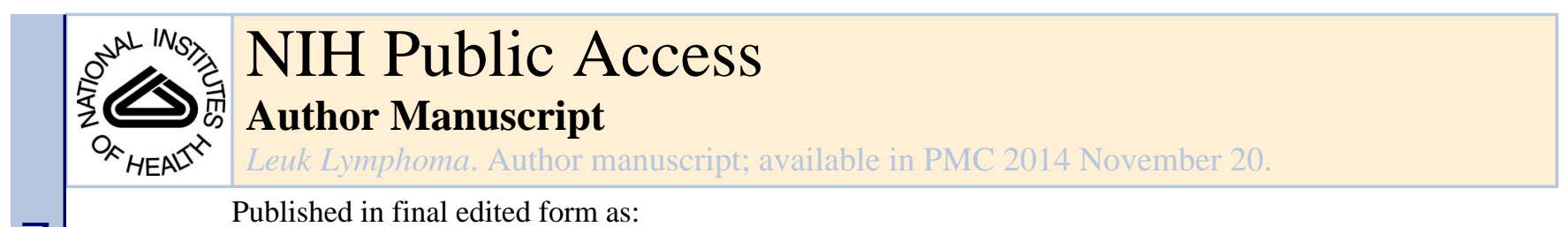

Published in final edited form as:

Leuk Lymphoma. 2011 January ; 52(1): 137-141. doi:10.3109/10428194.2010.524328.

\title{
Fludarabine, cyclophosphamide and antithymocyte globulin as total body irradiation-free conditioning for matched related and unrelated allogeneic stem cell transplantation in severe aplastic anemia
}

\author{
Paolo Anderlini ${ }^{1}$, Sandra Acholonu ${ }^{1}$, Grace-Julia Okoroji ${ }^{1}$, Roland E. Bassett $\mathrm{Jr}^{2}$, Sergio \\ Giralt ${ }^{1}$, Elizabeth J Shpall ${ }^{1}$, Muzaffar Qazilbash ${ }^{1}$, Uday Popat ${ }^{1}$, Laura Worth ${ }^{3}$, and Richard \\ E. Champlin 1 \\ ${ }^{1}$ Department of Stem Cell Transplantation and Cellular Therapy, The University of Texas M. D. \\ Anderson Cancer Center, Houston, Texas \\ ${ }^{2}$ Department of Biostatistics, The University of Texas M. D. Anderson Cancer Center, Houston, \\ Texas
}

${ }^{3}$ Department of Pediatrics, The University of Texas M. D. Anderson Cancer Center, Houston, Texas

\section{Abstract}

Twenty severe aplastic anemia (SAA) patients underwent allogeneic stem cell transplantation (allo-SCT) with fludarabine (FLU), cyclophosphamide and antithymocyte globulin from a matched related ( $n=7$, age $\geq 40$ ) or unrelated donor ( $n=13$, any age). Median age was 34 years (range 1-59). Median time from diagnosis to allo-SCT was 12 months (range 2-244). Seventeen out of 19 evaluable patients engrafted (90\%). There were two secondary graft failures (10\%). Median time to neutrophil recovery was 15 days (range 8-30). Chimerism studies indicated $290 \%$ donor-derived engraftment in 16/19 evaluable patients (75\%). Four out of 20 patients (20\%) developed acute (grade II-IV) GVHD, and 6/16 evaluable patients (37\%) developed chronic GVHD. We observed EBV reactivation and viremia in seven patients, which was successfully treated with rituximab in all but one instance (where it was self-limiting). Thirteen patients (62\%) are alive (including eight of the last nine treated) with a median follow-up of 30 months (range 3112). Seven patients expired (graft rejection $n=1$, GVHD $n=1$, multiorgan failure $n=1$, infection $\mathrm{n}=2$, EBV post-transplant lymphoproliferative disorder/PTLD $\mathrm{n}=2$ ). Total body irradiation-free, FLU-based conditioning for matched related and unrelated allo-SCT is feasible with high engraftment rates. EBV PTLD remains a drawback of this approach.

Correspondence: Paolo Anderlini, M.D., The University of Texas M. D. Anderson Cancer Center (UT-MDACC), Department of Stem Cell Transplantation and Cellular Therapy, 1515 Holcombe Boulevard - Unit 423, Houston, Texas 77030-4009, Phone: (713) 792-8750, Fax: (713) 794-4902. panderli@ mdanderson.org.

Authorship and Disclosures

PA designed the study and prepared the manuscript; SA, GJO provided data management; SA, GJO, REB performed the statistical analysis; all authors participated in the data interpretation and reviewed and approved the final manuscript.

The authors reported no potential conflict of interests. 


\section{Keywords}

Aplastic anemia; bone marrow failure; stem cell transplantation; bone marrow transplantation; conditioning regimen; fludarabine

\section{Introduction}

In matched unrelated (or alternative) donor (MUD) allogeneic stem cell transplantation (allo-SCT) for severe aplastic anemia (SAA), total body irradiation (TBI) is often employed to maximize engraftment and minimize graft rejection, although its toxicities are well known ${ }^{1,2}$. Dose optimization of the TBI component for MUD allografting was reported by Deeg et $\mathrm{al}^{2}$, showing that a TBI dose as low as $200 \mathrm{cGy}$ of TBI can ensure adequate engraftment. However, even at low doses, TBI can cause serious short-term toxicities, particularly diffuse pulmonary injury ${ }^{2}$. Moreover, long-term toxicities, such as growth retardation, secondary malignancies and hypothyroidism are also an important consideration, particularly in younger patients. Therefore, an effective and well-tolerated TBI-free conditioning regimen would be highly desirable. In 2005, Bacigalupo et al ${ }^{3}$ published their experience in 38 SAA patients who underwent an alternative donor allograft between 1998 and 2004 with a fludarabine (FLU), cyclophosphamide (CY) and antithymocyte globulin (ATG) conditioning regimen. The graft failure rate was $18 \%$, and the actuarial 2-year survival was $73 \%$. The authors suggested that this approach was particularly well suited for younger patients (age up to 14 years), due to a lower graft rejection rate in this cohort. Other groups have published similarly encouraging results in this setting 4,5 .

Similarly, in matched related donors (MRD), outcome in older (i.e. over age 40) SAA patients with conventional high-dose cyclophosphamide (CY) \pm ATG is generally felt to be poorer due to graft rejection, GVHD and, to a lesser extent, organ failure ${ }^{6,7}$. Recently fludarabine has been introduced in the conditioning regimen for MRD transplants as well. In 2006, Srinivasan et $\mathrm{al}^{8}$ reported 13 SAA patients (median age 27 years) allografted with a $\mathrm{FLU}, \mathrm{CY} \pm \mathrm{ATG}$ regimen with encouraging results. More recently, Maury et $\mathrm{al}^{9}$ have summarized the European Group for Blood and Marrow Transplantation (EBMT) retrospective experience on 30 SAA patients over the age of 30 years and allografted with fludarabine-based conditioning (FLU-CY \pm ATG). The 5-year survival of this cohort was $77 \%$, with a trend towards a reduced incidence of primary graft failure. That said, it should be pointed out that some disagreement still exists in this area. Sangiolo et a ${ }^{10}$ reported 20 SAA patients (median age 47 years, range 40-63) who underwent MRD allograft with conventional high-dose CY-ATG conditioning and an overall long-term survival of $70 \%$. Their conclusion was that MRD allo-SCT can be successfully performed in patients older than 40 years. However, these twenty patients were allografted over an 18 -year period (1988-2006), therefore patient selection may well have played a significant role in these favorable results.

We have tested fludarabine in reduced-intensity conditioning regimens in a variety of hematologic malignancies ${ }^{11,12}$, and we have long been interested in extending this concept 
to a TBI-free, fludarabine-based approach to allografting from alternative (and related) donors in SAA. This report summarizes the 10-year experience at the M.D. Anderson Cancer Center (MDACC) with TBI-free, fludarabine-based conditioning in SAA.

\section{Patients and Methods}

All consecutive patients with severe acquired aplastic anemia who underwent allogeneic SCT during a 10-year period (1999-2009) at the University of Texas M. D. Anderson Cancer Center Hospital (UT-MDACC) following conditioning with fludarabine, cyclophosphamide and ATG/thymoglobulin on a clinical protocol or on a compassionate basis are included in this report. Patients transplanted for other forms of marrow failure (Fanconi anemia, pure red cell aplasia, Diamond-Blackfan anemia, dyskeratosis congenita, etc.) were excluded. While initially this approach was limited to patients undergoing matched unrelated donor (MUD) transplants, since 2002 it was extended to matched related donor (MRD) transplants, provided patients were $\geq 40$ years old. This study was approved by the UT-MDACC Institutional Review Board (IRB), and all patients or their legal guardian provided written informed consent.

Over the 10-year period, doses and schedule for FLU, CY and ATG varied (Table 1). The regimen followed most consistently ( $\mathrm{n}=13$; patient \#1 to patient \#13 in the Table) as part of a prospective clinical pilot study (ClinicalTrials.gov NCT00427336) was FLU (30 mg/m sq IV $\times 4$ days), CY (300 mg/m sq IV x4 days), and thymoglobulin (THY, $3.75 \mathrm{mg} / \mathrm{kg}$ IV $\times 4$ days), in keeping with the report from Bacigalupo et $\mathrm{al}^{3}$. The duration of THY was reduced in early 2007 to three days in MRD recipients after a fatal case of Epstein-Barr virus (EBV)associated post-transplant lymphoproliferative disorder (EBV-PTLD) was diagnosed (Patient \#3). At the same time peripheral blood EBV viremia monitoring by PCR during the first 100 days post-transplant was introduced in the study, while prior to that it was carried out at the treating physician's discretion. Patient \#9 and \#12, as well patient \#11 had their four-day THY course reduced to 3 ands 2 days respectively for thymoglobulin intolerance $(n=2)$ and attending physician request $(n=1)$, respectively. Patient \#13 had her FLU dose reduced because of chronic renal failure. Doses and schedule for the other seven patients are listed in Table 1. These patients were transplanted on a compassionate, off-study basis (e.g. active uncontrolled infection, poor organ function, failure to meet other eligibility criteria), as well as before the study was open, or under a separate pediatric treatment plan (or both).

Demographics and characteristics of the twenty SAA patients are displayed in Table 1 . The median age at transplant for the whole group was 34 years (range 1-59). With the exception of patient \#8 (see below) all patients had acquired SAA. Median age for MUD and MRD allografts was 20 years (range 1-59) and 51 years (range 33-57), respectively. Seven pts $(33 \%)$ were $\geq 40$ years old. The donor was a MUD donor $(n=13)$ or a MRD donor $(n=7)$. Median time from diagnosis to allo-SCT was 12 months (range 2-244). As shown in Table 2 , bone marrow was used as stem cell source in all but four patients. Three patients received PBPCs and in one case umbilical cord blood was used. The median CD34+ cell dose infused was $4.10 \times 10^{6} / \mathrm{kg}$ (range $1.14-12.06$ ) for 18 pts with available data. Engraftment was defined as the first of three consecutive days of an absolute neutrophil count $2500 / \mu \mathrm{L}$. 
Platelet recovery was defined as the first of seven consecutive days of an unsupported platelet count $\geq 20,000 / \mu \mathrm{L}$.

Patients \#4 and \#6 had inadequate organ function (congestive heart failure and poor pulmonary function, respectively). Patient \#8 was transplanted for graft rejection and lifethreatening transfusion-dependent pancytopenia following an autologous-SCT for lymphoma. Patient \#13 was on chronic hemodialysis due to lupus nephritis-induced endstage renal disease. Patients \#5, \#7 and \#12 had active or presumed active infections at the time of transplant (cellulitis, fever of unknown origin, fungal pneumonia, respectively). Three patients included in this study (\#2,\#7 and \#10) were also included in the manuscript from Maury et al ${ }^{9}$.

\section{Results}

Hematological recovery data are outlined in Table 1 . Seventeen out of 19 evaluable patients engrafted (90\%). There were two secondary graft failures (10\%), defined as graft failure following initial engraftment. Median time to neutrophil recovery was 15 days (range 8-30). Median time to platelet recovery was 15 days (range 9-73) for 17 evaluable patients. Chimerism studies indicated $\geqq 90 \%$ donor-derived engraftment in $16 / 19$ evaluable patients (75\%). In 13/19 evaluable patients (68\%) there was complete donor-derived engraftment. Two patients (10\%) experienced secondary graft rejection. In addition to two (fatal) EBVPTLD cases (see below), we observed EBV reactivation and viremia in seven patients, which was successfully treated with rituximab in all but one instance (where it was selflimiting).

Four out of 20 evaluable patients (20\%) developed acute (grade II-IV) graft-versus-host disease (GVHD), and 6/16 evaluable patients (37\%) developed chronic GVHD. Patient outcome data are presented in Table 2 . Thirteen patients (62\%) are alive (including eight out the last nine treated) with a median follow-up of 30 months (range 3-113). Seven patients expired (MUD $n=5$, MRD n=2). Causes of death included graft rejection $n=1$, infection $n=2$, GVHD n=1, multiorgan failure $\mathrm{n}=1, \mathrm{EBV}$-associated post-transplant lymphoproliferative disorders (EBV-PTLD) $n=2$. In one of the two EBV-PTLD cases (patient \#3), a fine needle aspirate of a cervical node (carried out prior to rituximab therapy) showed large atypical lymphoid cells, some of which demonstrated plasmacytoid morphology. Flow cytometry showed the atypical cells to be positive for CD19 dim, CD20 partial, and CD138 partial (a plasma-cell associated antigen), with dim surface immunoglobulin light chain expression.

There was one early death (defined as death prior to day 30) and no known late graft failures. Overall 2-year survival (actuarial estimate) for the whole group $(\mathrm{n}=20)$ and for the thirteen patients (MRD n=7; MUD $\mathrm{n}=6$ ) treated according to Bacigalupo et $\mathrm{al}^{3}$ are $61 \%$ (95\% CI 42\%-89\%) and 82\% (95\% CI 62\%-100\%), respectively (Figure 1, A and B).

\section{Discussion}

This report describes the 10-year experience at UT-MDACC with fludarabine, cyclophosphamide and antithymocyte globulin as total body irradiation-free conditioning for matched related and unrelated allogeneic stem cell transplantation in severe acquired 
aplastic anemia. The idea of combining fludarabine and alkylating agents for synergism in the treatment of hematologic malignancies was originally pioneered at UT-MDACC ${ }^{13}$. However, to our knowledge, it was Bacigalupo et $\mathrm{al}^{3}$ who adopted this approach in reducedintensity conditioning for matched unrelated allo-SCT in SAA. The concept of a TBI-free conditioning regimen in this setting is undoubtedly quite appealing, in view of the shortterm and long-term toxicities associated with radiation exposure, particularly in younger patients ${ }^{1,2}$. That being said, graft rejection has been a potential concern, so the addition of at least low-dose TBI has recently been advocated ${ }^{14}$. Similarly, the same group of investigators has proposed the use of reduced-intensity, fludarabine-based conditioning in allo-SCT from MRD for SAA in older (>30 yrs) recipients ${ }^{9}$. Initial results appear quite encouraging.

We readily and fully acknowledge the many limitations of our study. They include a small sample size, a mix of matched related and unrelated donors, possible selection bias, a long time interval (ten years) evaluated while supportive care and HLA typing and matching techniques were evolving rapidly, as well as some heterogeneity in the patients treated and preparative regimens employed. We are also quite aware that, because of these reasons, comparing these data with the current literature on this topic would be challenging. The seemingly better outcome of the latest patients treated defies an immediate explanation. It could be accounted for by patient selection, more accurate HLA typing and matching as well as improved supportive care.

Still, we believe this study suggests that FLU-based, TBI-free conditioning for matched related and unrelated allo-SCT is feasible, with high engraftment rates and low early mortality. While we did not observe late graft failures, this complication has been reported and the sample size and follow-up of this study are inadequate to be reassuring about this issue. Presumably due to high-dose thymoglobulin, GVHD-related incidence and mortality was low ${ }^{15}, 16$, although EBV-PTLD was a drawback of this approach ${ }^{17}$. Interestingly, the lymphoid proliferation in one of the two EBV-PTLD cases we observed was only weakly CD20-positive, casting some doubts on the efficacy of rituximab in some of these patients. Similar CD20-negative cases have been reported in the literature ${ }^{18}$, 19 . Prevention as well as effective treatment of EBV-PTLD remains a priority and a challenge, and new strategies are clearly needed ${ }^{20}$.

We also believe these data as a whole do support the concept that fludarabine can be a key component of the conditioning regimen for allografting in SAA. Its appropriate role in related donor transplants, and whether low-dose TBI is indeed required in the alternative donor setting (particularly in younger patients) deserve further, and ongoing, investigation ${ }^{14}$.

\section{Acknowledgments}

The Authors would like to thank the UT-MDACC nursing staff, physician's assistants, transplant coordinators, data managers and case managers (in particular Mrs. Elvira Cruz). We also would like to thank Mrs. Gloria McCormick for her role as research nurse and Mrs. Esthela U. Guillen for her secretarial assistance. 


\section{References}

1. Deeg HJ, Amylon ID, Harris RE, Collins R, Beatty PG, Feig S, et al. Marrow transplants from unrelated donors for patients with aplastic anemia: minimum effective dose of total body irradiation. Biol Blood Marrow Transplant. 2001; 7(4):208-215. [PubMed: 11349807]

2. Deeg HJ, O'Donnell M, Tolar J, Agarwal R, Harris RE, Feig SA, et al. Optimization of conditioning for marrow transplantation from unrelated donors for patients with aplastic anemia after failure of immunosuppressive therapy. Blood. 2006; 108(5):1485-1491. [PubMed: 16684959]

3. Bacigalupo A, Locatelli F, Lanino E, Marsh J, Socié G, Maury S, et al. for the Severe Aplastic Anemia Working Party of the European Group for Blood and Marrow Transplantation. Fludarabine, cyclophosphamide and anti-thymocyte globulin for alternative donor transplants in acquired severe aplastic anemia: a report from the EBMT-SAA Working Party. Bone Marrow Transplant. 2005; 36(11):947-950. [PubMed: 16205733]

4. Lee JH, Choi SJ, Lee JH, Lee YS, Seol M, Ryu SG, et al. Non-total body irradiation containing preparative regimen in alternative donor bone marrow transplantation for severe aplastic anemia. Bone Marrow Transplant. 2005; 35(8):755-761. [PubMed: 15735661]

5. Kang HJ, Shin HY, Choi HS, Ahn HS. Fludarabine, cyclophosphamide plus thymoglobulin conditioning regimen for unrelated bone marrow transplantation in severe aplastic anemia. Bone Marrow Transpl. 2004; 34(11):939-943.

6. Bacigalupo A. Aplastic anemia: pathogenesis and treatment. Hematology (Am Soc Hematol Educ Program). 2007:23-28. [PubMed: 18024605]

7. Gupta V, Carreras J, Bajorunaite R, Gale RP, Sabloff M, Aljurf M, et al. Hematopoietic Recovery and Overall Survival after HLA-Matched Sibling Transplants for Older Patients with Severe Aplastic Anemia (SAA). Blood. 2008; 112:2169. (abstr).

8. Srinivasan R, Takahashi Y, McCoy JP, Espinoza-Delgado I, Dorrance C, Igarashi T, et al. Overcoming graft rejection in heavily transfused and allo-immunised patients with bone marrow failure syndromes using fludarabine-based haematopoietic cell transplantation. Br J Haematol. 2006; 133(3):305-314. [PubMed: 16643433]

9. Maury S, Bacigalupo A, Anderlini P, Aljurf M, Marsh J, Socié G, et al. on behalf of the Severe Aplastic Anemia Working Party, European Group for Blood and Marrow Transplantation (EBMTSAAWP). Improved outcome of patients older than 30 years receiving HLA-identical sibling hematopoietic stem cell transplantation for severe acquired aplastic anemia using fludarabine-based conditioning: a comparison with conventional conditioning regimen. Haematologica. 2009; 94(9): 1312-1315. [PubMed: 19734425]

10. Sangiolo D, Storb R, Lsisenring W. Outcome of Allogeneic Hematopoietic Cell Transplantation (HCT) for Patients with Severe Aplastic (SAA) above the age of 40 Years. Blood. 2006; 108:3019. (abstr).

11. de Lima M, Anagnostopoulos A, Munsell M, Shahjahan M, Ueno N, Ippoliti C, et al. Nonablative versus reduced-intensity conditioning regimens in the treatment of acute myeloid leukemia and high-risk myelodysplastic syndrome: dose is relevant for long-term disease control after allogeneic hematopoietic stem cell transplantation. Blood. 2004; 104(3):865-872. [PubMed: 15090449]

12. Khouri IF, McLaughlin P, Saliba RM, Hosing C, Korbling M, Lee MS, et al. Eight-year experience with allogeneic stem cell transplantation for relapsed follicular lymphoma after nonmyeloablative conditioning with fludarabine, cyclophosphamide, and rituximab. Blood. 2008; 111(12):5530 5536. [PubMed: 18411419] acigalupo A, Dominietto A, Soracco M, Raiola AM, Van Lint MT, Lamparelli T, et al. Rituximab Prophylaxis EBV Reactivation after Alternative Donor Transplants Following Anti-Thymocyte Globulin-Based Conditioning Regimens. Blood. 2008; 112:2232. (abstr). [PubMed: 18617635]

13. O'Brien SM, Kantarjian HM, Cortes J, Beran M, Koller CA, Giles FJ, et al. Results of the fludarabine and cyclophosphamide combination regimen in chronic lymphocytic leukemia. J Clin Oncol. 2001; 19(5):1414-1420. [PubMed: 11230486]

14. Bacigalupo A, Locatelli F, Lanino E, Marsh J, Socie G, Passweg J. Fludarabine, Cyclophosphamide With or Without Low Dose TBI for Alternative Donor Transplants in Acquired Aplastic Anemia (SAA): A Report from the EBMT-SAA Working Party. Biol Blood Marrow Transplant. 2009; 15 Suppl 2(2) 
15. Deeg HJ, Storer B, Boeckh M, Martin PJ, McCune JS, Myerson D, et al. Reduced incidence of acute and chronic graft-versus-host disease with the addition of thymoglobulin to a targeted busulfan/cyclophosphamide regimen. Biol Blood Marrow Transplant. 2006; 12:573-584. [PubMed: 16635793]

16. Bacigalupo A, Lamparelli T, Barisione G, Bruzzi P, Guidi S, Alessandrino PE, et al. for the Gruppo Italiano Trapianti Midollo Osseo (GITMO). Thymoglobulin prevents chronic graft-versushost disease, chronic lung dysfunction, and late transplant-related mortality: long-term follow-up of a randomized trial in patients undergoing unrelated donor transplantation. Biol Blood Marrow Transplant. 2006; 5:560-565. [PubMed: 16635791]

17. Styczynski J, Einsele H, Gil L, Ljungman P. Outcome of treatment of Epstein-Barr virus-related post-transplant lymphoproliferative disorder in hematopoietic stem cell recipients: a comprehensive review of reported cases. Transpl Infect Dis. 2009; 11(5):383-392. [PubMed: 19558376]

18. Anderlini P, Valvuena JR, Champlin RE, Bueso-Ramos CE. Epstein-Barr virus-associated, CD20polyclonal lymphoproliferative disorder after matched unrelated donor marrow transplant. Bone Marrow Transpl. 2004; 34(10):919-921. (letter).

19. Kahl C, Martinez A. Moll S Post-transplant lymphoproliferative disorder of plasmacytoma-like subtype. Br J Haematol. 2007; 139(4):518. [PubMed: 17784882]

20. Bacigalupo A, Dominietto A, Soracco M, Raiola AM, Van Lint MT, Lamparelli T, et al. Rituximab Prophylaxis EBV Reactivation after Alternative Donor Transplants Following AntiThymocyte Globulin-Based Conditioning Regimens. Blood. 2008; 112:2232. (abstr). [PubMed: 18617635] 


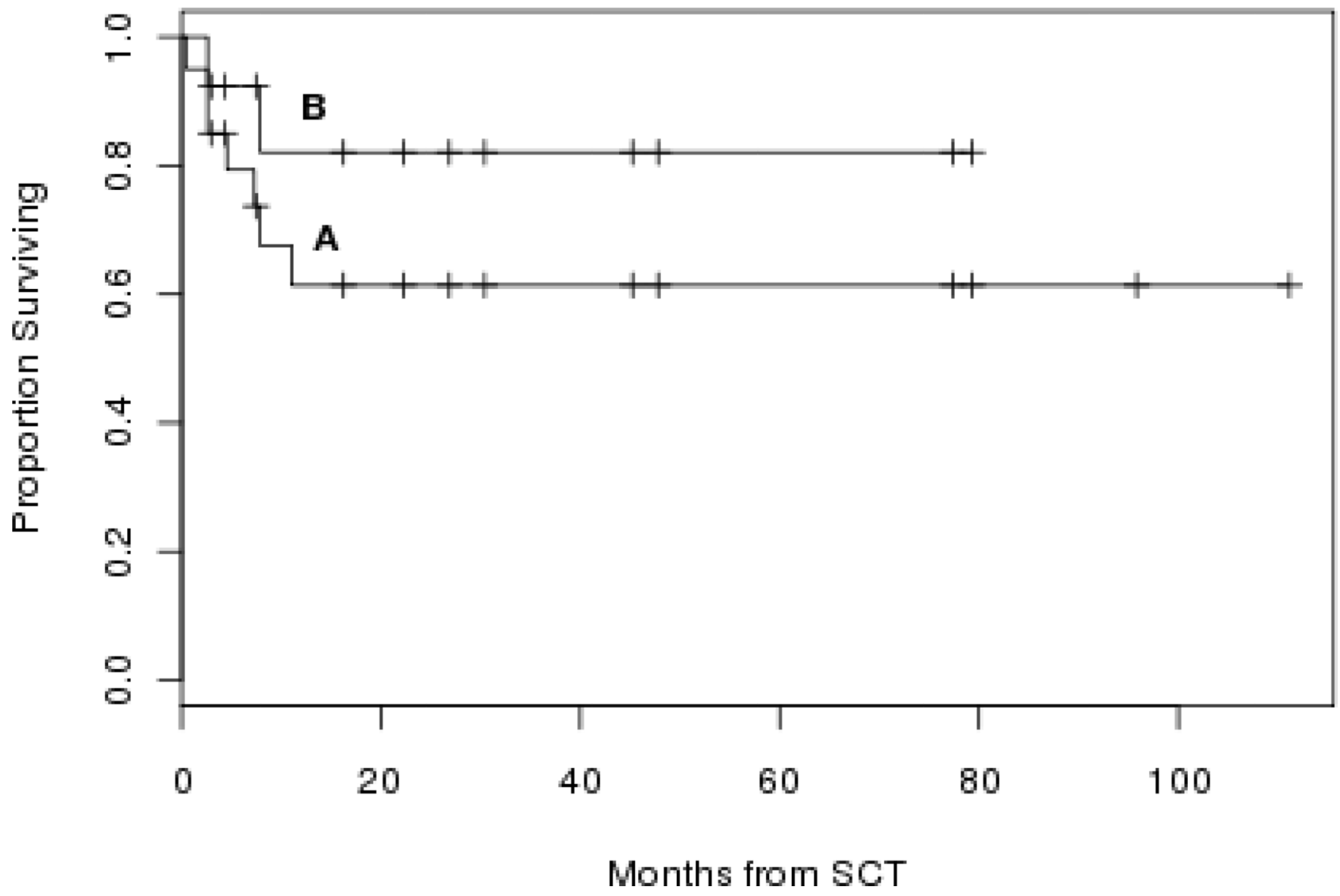

Figure 1.

Overall survival (actuarial estimate) for the whole group $(n=20)$ (curve A) and for the thirteen patients treated according to Bacigalupo et $\mathrm{al}^{3}$ (curve B). SCT: stem cell transplant. 


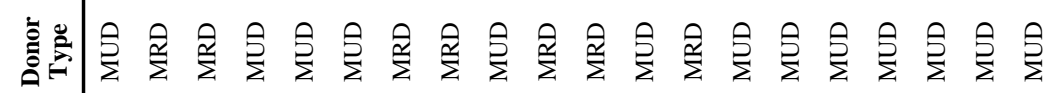

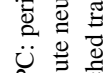

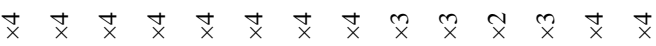

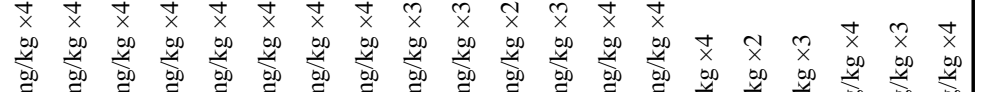

要

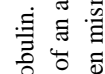

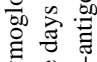

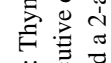

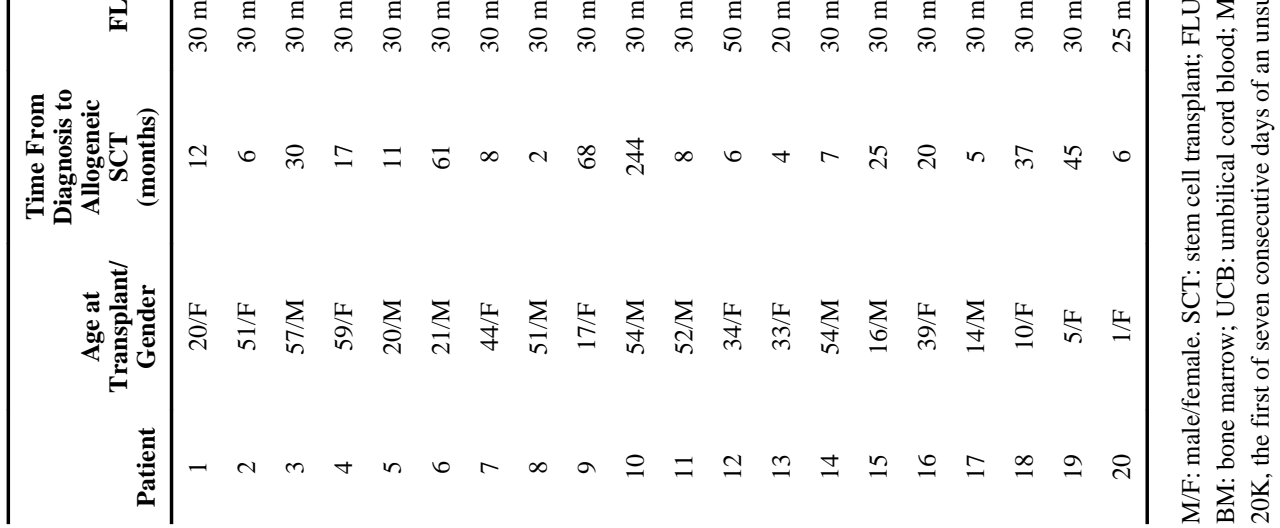




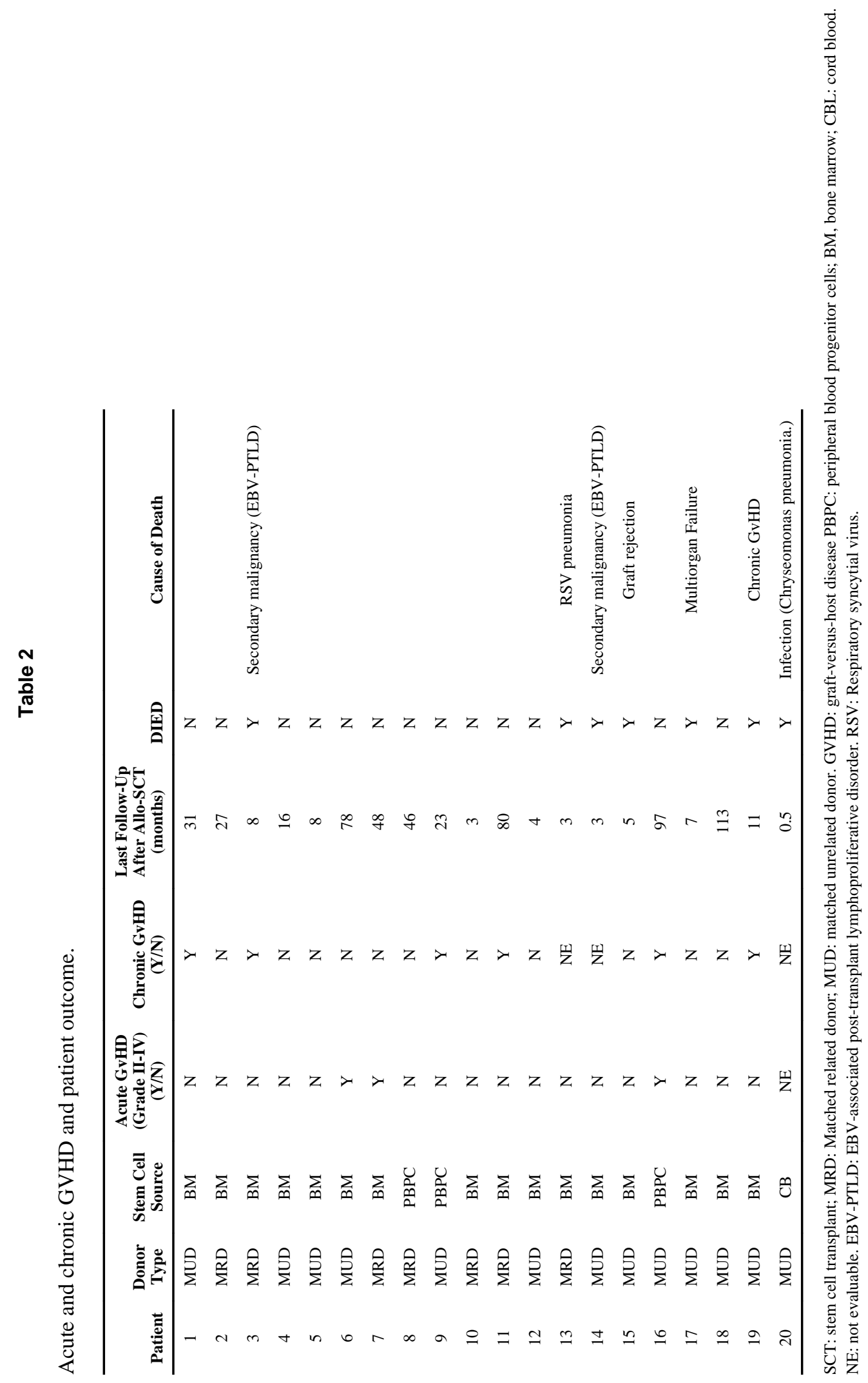

